\title{
ALTERATIONS OF ANTITUMOR AND METABOLIC RESPONSES IN L5178Y-R LYMPHOMA-BEARING MICE AFTER ONLY 30-MINUTE DAILY CHRONIC STRESS EXPOSURE
}

\author{
D. Caballero-Hernandez*, D. Najera-Valderrabano, A. Valadez-Lira, M. Franco-Molina, \\ R. Gomez-Flores, P. Tamez-Guerra, R. Tamez-Guerra, C. Rodríguez-Padilla \\ Department of Microbiology and Immunology, Laboratory of Immunology and Virology, \\ Faculty of Biological Sciences, Autonomous University of Nuevo León, \\ San Nicolás de los Garza, NL 66455, Mexico
}

\begin{abstract}
Aim: In stress research, reducing times of stress induction may contribute to improving the well-being of experimental animals, especially in cancer models, already under physiological distress. To support this idea, we evaluated the effects of a short-timed stress protocol on endocrine, metabolic and immune indicators in mice bearing the L5178Y-R lymphoma. Materials and Methods: A 30-minute daily stress protocol was applied for 28 days to healthy and lymphoma-bearing BALB/c mice; body weight, plasma levels of corticosterone, norepinephrine, Th1/Th2 cytokines, insulin, and leptin, were measured. Results: We found a $12 \%$ significant decrease in body weight in non-tumor bearing mice under stress $(p<0.007)$. The disruption of weight evolution was accompanied by a stress induced $85 \%$ decrease in plasmatic leptin $(p<0.01)$ and total reduction of insulin. Tumor burden alone was associated to an increase in more than two-fold of plasmatic levels of norepinephrine $(p<0.008)$. Neither stress nor tumor or their combination, resulted in an elevation of systemic IL-6. IFN- $\gamma$ levels were 20 times higher in lymphoma-bearing animals when compared with non-tumor bearing mice $(\boldsymbol{p}<\mathbf{0 . 0 1})$; however, under stress, this response was reduced by half, indicating a suppressing effect of chronic stress on the antitumor immune response. Conclusion: A short-timed stress induction is enough to cause significant alterations in the metabolism and immunity of healthy and tumor-bearing mice, supporting the use of short-timed protocols as an efficient way to induce chronic stress that also considers concerns regarding the well-being of experimental animals in biomedical research. Key Words: psychogenic stress, tumor models, immunosuppression, glucose homeostasis, IFN- $\gamma$, leptin, animal welfare.
\end{abstract}

Psychogenic stress has been proven to have a significant role in cancer initiation and progression; hence, the study of its many biological effects is of great interest [1, 2]. Besides providing a rational basis for preventive stress management, such studies may identify targets for therapeutic intervention aiming at improving quality of life in cancer patients. In this sense, an often used pre-clinical approach to study the influence of stress on cancer involves the application of a stress-inducing paradigm in tumor-bearing animals [3]. One of the most frequently used is the restraint stress paradigm, which involves placing mice or rats in a small, closed container to reduce its ability to move $[4,5]$. Other paradigms, such as forced swim and social isolation are also valuable to dissect the mechanisms behind the biological effects of psychogenic stress [6, 7].

These stress-inducing paradigms must be able to induce the activation of the hypothalamic-pituitaryadrenal (HPA) axis through the secretion of cortisol, and/or the sympathetic adrenal medullary (SMA) axis with the release of noradrenaline; both hormones physiological markers of stress [5]. More recently, the IL6-STAT3 axis has been revealed as a pathway also involved in mediating the effects of psychogenic stress, where systemic IL- 6 elevation has been associated with stressful conditions [8], with the potential to affect a diverse array of cellular and molecular targets [9].

Submitted: September 8, 2017.

*Correspondence: E-mail: diana.caballerohr@uanl.edu.mx Abbreviations used: HPA - hypothalamic-pituitary-adrenal; NE norepinephrine; SAM - sympathetic adrenal medullary.
Other markers of chronic stress activation also include body weight fluctuations and changes in behavior, including depression and feeding behavior [10]. Interestingly, cancer, as a form of physiological stress, can also elicit these same responses [11].

In the context of the increasing concern regarding animal well-being in biomedical research, for psychogenic stress research, the induction of stress makes necessary to cause a certain level of discomfort in experimental animals, but how much and for how long is not a settled matter, although efforts have been made to optimize times of induction and parameters [12]. For chronic stress induced by restriction of movement, we have found that a significant number of studies employ the restraint paradigm for periods ranging from 2 to $6 \mathrm{~h}$ [4]. Previously, Zamora-González [13] had demonstrated that a daily protocol of $30 \mathrm{~min}$ of restraint stress, that also includes the aleatory use of the forced swim test, as a stress inductor, and resting days to avoid habituation in animals, is enough for the activation of the HPA axis in BALB/c mice. In this work, we applied this shorttimed stress inducing protocol to test its ability to elicit a chronic stress response in the L5178Y-R mouse model of lymphoma, with the aim of establishing an effective mouse model to study cancer initiation and progression under psychogenic stress and that is also compliant with animal welfare concerns regarding the use of experimental animals in biomedical research.

\section{MATERIAL AND METHODS}

Animals. Female, 10-12-week-old BALB/c mice were purchased from Harlan Laboratories S.A. de C.V. 
(México City, Mexico) and were housed in an animal care facility at $24-26{ }^{\circ} \mathrm{C}$ with $45 \%$ humidity and a $12 \mathrm{~h}$ light: $12 \mathrm{~h}$ dark cycle, in individually ventilated cages with environment enrichment; water and food were provided ad libitum. All procedures performed in animals followed ethical standards on animal welfare and were approved by the Institutional Animal Care and Use Committee (CICUAL-LIV).

L5178Y-R lymphoma model. To test the influence of a chronic stress paradigm on tumor bearing animals we used the L5178Y-R lymphoma mouse model. This lymphoma is maintained in ascites form in BALB/c mice by the intraperitoneal transplantation of $1 \cdot 10^{6} \mathrm{~L} 5178 \mathrm{Y}-\mathrm{R}$ cells per mouse. Thirteen days after inoculation, ascites is collected from the peritoneal cavity of mice killed by cervical dislocation under anesthesia. The cell suspension is then washed twice in PBS by centrifuging at $2000 \mathrm{rpm}$ for $10 \mathrm{~min}$, finally adjusting to $2 \cdot 10^{7}$ cells $/ \mathrm{ml}$ in sterile PBS. To induce the formation of a solid tumor, $0.1 \mathrm{ml}$ of the cell suspension was inoculated in the right flank of mice by intramuscular injection [14].

Chronic stress protocol. Animals were randomly distributed into 4 experimental groups $(n=4)$ : non-tumor bearing animals in resting conditions (a) and under stress (b); and lymphoma-bearing animals in resting conditions (c) and under stress (d). Animals in resting conditions remained in their cages while the stress sessions were carried on. Experimental mice were subjected to a 28 days chronic stress protocol consisting in two stress paradigms [13]: restraint stress ( $30 \mathrm{~min}$ ) and forced swim ( $15 \mathrm{~min}$ ); these were randomly applied from day 0 , as shown in Fig. 1. For restraint stress, the animals were placed in $50 \mathrm{ml}$ conical tubes with ventilation holes. Asecond, larger container was employed for tumor-bearing animals once tumor size made difficult to contain the animals in the conical tubes. For the forced swim test, a plastic cylindrical tank, $19 \mathrm{~cm}$ height by $15 \mathrm{~cm}$ diameter, was employed; the container was filled up to $8.5 \mathrm{~cm}$ from the bottom with tap water, temperature was setat $25^{\circ} \mathrm{C}$. Mice, one at a time, were carefully placed in the water under constant vigilance, and after $15 \mathrm{~min}$, were removed and gently dried before being returned to the cage.

On day 4 , mice were inoculated with the L5178Y-R lymphoma, as previously described. On days 9, 16 and 23 the anhedonia test was performed. Body weight was measured every 4 days. At the end of the protocol, blood was collected by cardiac puncture of mice under anesthesia, after which animals were euthanized by cervical dislocation and tumor and liver samples were collected and stored frozen. Blood was centrifuged at $5000 \mathrm{rpm}$, and plasma was collected and stored at $-80^{\circ} \mathrm{C}$ until analysis.

Anhedonia test. To assess behavioral disruptions caused by the stress protocol, animals were subjected to the sucrose preference test, also known as anhedonia test. This test measures the animal's preference for sweetened drinks, a reward, over plain water, as an indicator of the ability to feel pleasure. The test was carried out in the housing cages, two drink bottles per cage, one containing $200 \mathrm{ml}$ of tap water, and the other the same volume of a $2 \%$ sucrose solution (Jalmek Científica, S.A. de C.V.) were made available to the animals for $48 \mathrm{~h}$. Water and sucrose solution intake were measured at 24 and $48 \mathrm{~h}$. Sucrose preference was calculated as the percentage of sucrose solution intake over total fluid intake [15]. Before testing, the animals were habituated to having the 2 bottles, water and sucrose solution, over a 3 day period, switching the position of the bottles every $24 \mathrm{~h}$ [16], to avoid potential bias due to the rejection of a new item by the animals.

Cytokines and hormones. Plasma levels of IL-2, IL-4, IL-6, IFN- $\gamma$, TNF, IL-17A, and IL-10 were determined in an Accuri 6 flow cytometer using the Cytometric Bead Array Mouse Th1/Th2/Th17 kit from BD Biosciences (San Jose, CA), data was analyzed with the FCAP Array Software V 3.0 (Soft Flow Hungary, Ltd., Pécs, Hungary). Plasma levels of corticosterone and norepinephrine were measured with the Corticosterone EIA Kit from Enzo Life Sciences (NY, USA) and the Mouse Noradrenaline ELISA kit from MyBiosource (San Diego, California), respectively. Insulin and leptin were measured in a MAGPIX ${ }^{\circledast}$ system using the Mouse Metabolic Magnetic Bead Panel from Merck Millipore.

Statistical analysis. Level of significance was assessed by Student's $t$ test. Statistical analyse were performed by using the website for statistical computation VassarStats, freely available at $h$ ttp://vassarstats.net/.

\section{RESULTS}

We subjected non-tumor bearing and lymphomabearing mice to a short-timed stress paradigm for 28 days (Fig. 1); we found that this chronic short stress induction was sufficient to significantly alter some key, but not all, immunological and metabolic parameters evaluated. Despite being brief, the stress protocol interfered with weight gain in non-tumor and lymphomabearing mice. In Fig. 2, the weight progression of experimental animals during the experiment is shown. By the end of the protocol, at day $28^{\text {th }}$, non-bearing tumor animals in resting conditions showed a $9 \%$ mean increment of their weight, meanwhile, stressed mice not only failed to gain weight, at day 28 their mean weight was $3 \%$ lower than on day 0 of the experiment $(p<0.007)$. For lymphoma-bearing mice, weight gain in stressed animals was half of those resting (16\%), here; the increasing weight displayed by lymphomabearing animals must be attributed to tumor growth, having the unintended effect of masking weight loss by the animals.

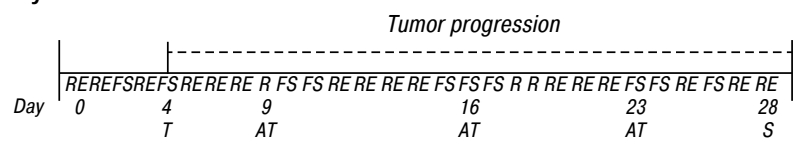

Fig. 1. Stress protocol timeline. Female BALB/c mice, 11 week-old at the beginning of the experiment, were subjected to a chronic stress protocol combining the restraint and forced swim paradigms for 28 days. Control animals were kept undisturbed in their cages. RE - restraint stress; FS - forced swim; $\mathrm{R}$ - resting; $\mathrm{T}$ - tumor inoculation; AT - anhedonia test; $\mathrm{S}$ mice sacrifice and sample collecting 


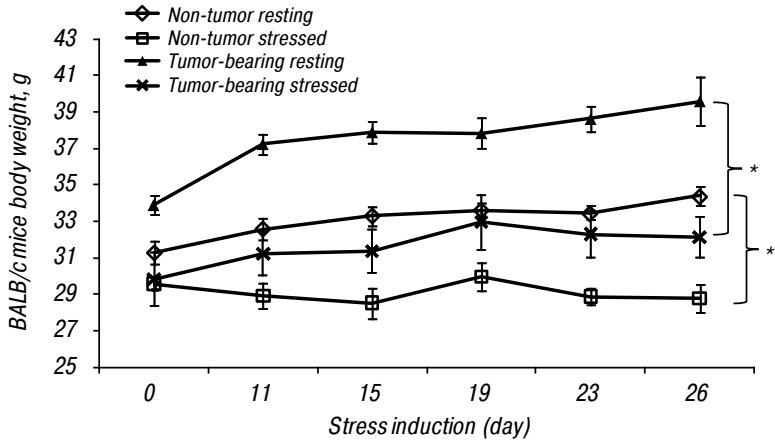

Fig. 2. Body weight evolution under chronic stress. Body weight was measured every 3 days. Weight gain is expressed as a percentage of the weigh at the beginning of the experiment. Data represent mean \pm SEM of 4 animals from a representative experiment. ${ }^{*} p<0.05$ as compared with the respective control

To evaluate the effect of chronic stress on the L5178Y-R model, the anhedonia test was performed twice during the experiment; for $48 \mathrm{~h}$, animals were offered the choice between two bottles, one containing tap water and the other a $2 \%$ sucrose solution, a decrease in sucrose solution intake would be indicative of depression in the animals, however, no differences were detected among the experimental groups, finding sucrose preference across the groups ranging from $76-81 \%$, above the $65 \%$ used as criterion for anhedonia [16].

To test the activation of the HPA and adrenergic system, the plasmatic levels of corticosterone and norepinephrine were measured using ELISA. According to the results, the short induction of stress used in this study failed to activate the HPA system, levels of corticosterone remained unchanged between the experimental groups (Table). As for the adrenergic system, although norepinephrine levels were unaffected by stress, a $240 \%$ increase was observed for resting lymphoma-bearing animals when compared to resting non-tumor bearing animals $(p<0.008)$, suggesting that tumor burden, but not the stress protocol tested, activates the sympathetic system in BALB/c mice (Table).

Table. Neuroendocrine responses of female BALB/c mice to chronic stress

\begin{tabular}{clcc}
\hline \multicolumn{1}{c}{ Mice } & Groups & Corticosterone, $\mathrm{pg} / \mathrm{ml}$ & Norepinephrine, $\mathrm{pg} / \mathrm{ml}$ \\
\hline Non-tumor & Resting & $111.5 \pm 8.7$ & $131.3 \pm 34.6$ \\
& Stress & $124.5 \pm 4.7$ & $163.8 \pm 118.0$ \\
Tumor & Resting & $120.3 \pm 6.1$ & $315.2 \pm 88.2$ \\
& Stress & $138.5 \pm 6.8$ & $262.8 \pm 78.0$ \\
\hline
\end{tabular}

Plasma levels of Th1, Th2 and Th17 cytokines were also evaluated. Here, we found that although IL-4, IL-2 and TNF were detected (concentration ranging from $8.5-10 \mathrm{pg} / \mathrm{ml}$ ), no differences were observed among the experimental groups. Unexpectedly, IL-6 was not detected in any experimental group. Then, according to the results, anti and pro-inflammatory cytokines were mostly unaffected by the short time of stress protocol tested, with one important exception, levels of IFN- $\gamma$ were significantly elevated in lymphoma-bearing mice $(40.06 \pm 9 \mathrm{pg} / \mathrm{ml})$ when compared to non-bearing tumor animals, however lymphoma-bearing animals under chronic stress showed $50 \%$ lower levels of plasmatic IFN- $\gamma(p<0.05)$ (Fig. 3).

Non-tumor bearing animals showed a reduction of $95.8 \%$ in levels of plasmatic leptin in animals under stress $(p<0.01)$. In lymphoma-bearing animals, levels of leptin were also lower when compared to nontumor bearing animals, regardless of stress induction (Fig. 4). Although insulin was detected in non-tumor bearing animals $(236 \pm 160 \mathrm{pg} / \mathrm{ml})$, it was not detected in non-tumor bearing animals under stress, or in resting or stressed lymphoma-bearing mice.

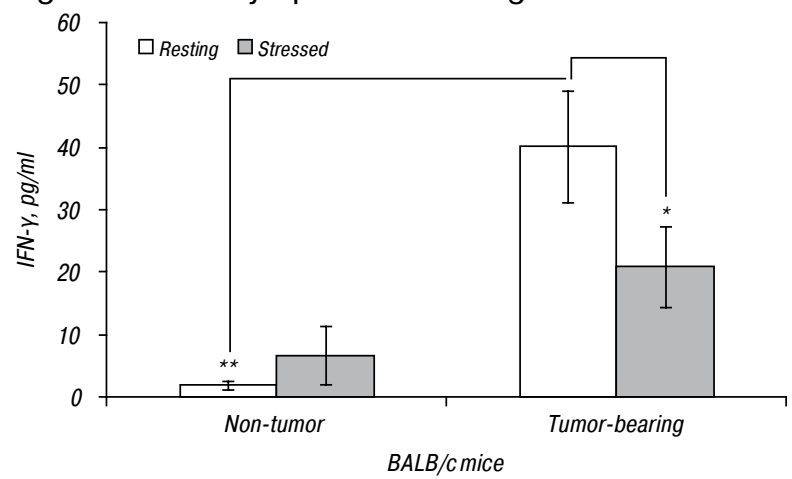

Fig. 3. Plasmatic levels of IFN- $\gamma$ in BALB/c mice under chronic stress. At day 28, animals were anesthetized with ketamine/ xylazine and whole blood was collected by terminal cardiac puncture, sample collection was performed between 16:00 and 20:00 hours. Levels of IFN- $\gamma$ were measured by flow cytometry using a cytometric bead array. Data represent mean \pm SEM of 4 animals from a representative experiment. ${ }^{*} p<0.05$, ${ }^{* *} p<0.01$ as compared with the respective control

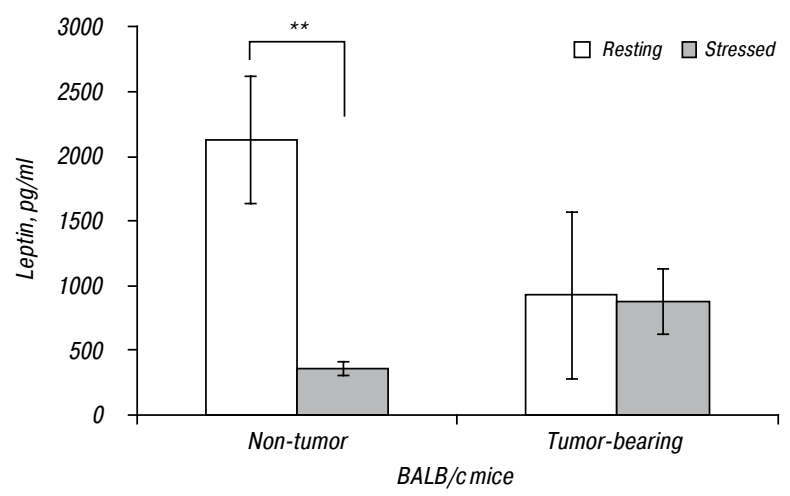

Fig. 4. Plasmatic levels of leptin in BALB/c mice under chronic stress. At day 28 , animals were anesthetized with ketamine/ xylazine and whole blood was collected by terminal cardiac puncture, sample collection was performed between 16:00 and 20:00 hours. Levels of leptin were measured by multiplexed immunoassay in a MAGPIX ${ }^{\circledR}$ system. Data represent mean \pm SEM of 4 animals from a representative experiment. ${ }^{* \star} p<0.01$ as compared with the respective control

\section{DISCUSSION}

In this work, with the aim of establishing a refined model to measure the effects of psychogenic stress in cancer, considering the welfare of experimental animals, we measured the effects of a short-timed chronic stress protocol in mice bearing the L5178Y-R lympho$\mathrm{ma}$. We found that although not all parameters were affected, some were distinctly altered by the stress protocol; with some of the observed changes suggesting a disruption in the ability to maintain body weight that is potentially being driven by adrenergic activation. 
Body weight is a useful and inexpensive parameter to assess the effect of stress on living beings under various conditions. In this work, under stress, the body weight of non-tumor bearing animals was significantly lower than in resting non-tumor bearing mice, this disruption could also be observed in lymphoma-bearing mice. Weight loss is an indication of a compromised energy metabolism in the animals, it can also be the result of behavioral changes, such as decreasing food intake, however we have no data to support this hypothesis and the results of the anhedonia test (data not shown) do not suggest that this short-timed stress induction paradigm is enough to change the behavior of animals.

The sustained activation of both the HPA axis and adrenergic pathways during chronic stress may trigger adaptive thermogenesis with the mobilization of the body energy stores leading to weight loss. Under stress, changes in feeding behavior could also be involved in weight loss. These changes may be attributed to HPA activation, however, according to our results, the protocol tested was not able to activate the HPA axis; levels of corticosterone were not different between resting and stressed animals. This contradicts the findings of Zamora-González et al. [13] since they reported the elevation of corticosterone in animals subjected to the same stress protocol. Given that they used mice of the same strain, sex, and age, these contradicting results may be explained by the time of the day the sample was collected. Overall, there is ample controversy regarding HPA axis activation as an indicator of stress induction, since a significant number of studies have failed to find alterations in corticosterone levels in animals under restraint and social stress [17].

Another potential contributor to weight loss is the elevation of peripheral IL-6, a pro-inflammatory cytokine that has been linked to the induction of uncoupling-1 protein leading to brown tissue activation and weight loss [18], however, as previously stated in the results section, this cytokine was mostly absent in plasma in experimental animals, regardless of treatment. As for adrenergic activation, lymphoma-bearing animals showed elevated plasma levels of norepinephrine, and although non-tumor bearing animals under stress also showed an elevation when compared to resting animals, it was not statistically significant. Weight decrease in non-tumor bearing animals under stress, may be in part due to adrenergic activation.

The analysis of plasmatic levels of leptin and insulin supports the view of a disrupted metabolism in the animals, caused not only by stress but also by tumor burden, that may be involved in weight maintenance. In non-tumor bearing animals under stress, levels of leptin were significantly lower, $95.8 \%$, than in resting animals. Levels of leptin were not different in L5178Y-R lymphoma-bearing mice under stress when compared with resting animals. It could be expected that changes in levels of leptin, a satiety messenger, to be accompanied by changes in food intake behavior, playing a role in weight maintenance, something that needs to be assessed. Insulin was absent in non-tumor bearing animals under stress, and in lymphoma-bearing mice, under resting and stress conditions as well.

In regard to IFN- $\gamma$, it was evident that tumor burden causes an elevation in plasmatic IFN- $\gamma$ in the animals; this change is consistent with an antitumoral response mounted by the host. However, such a response was reduced in lymphoma-bearing mice subjected to stress by half. Previously, it has been demonstrated that acute and chronic stress by restraint or immobilization induces a decrease in INF- $Y$ plasma levels in rats $[19,20]$ and in a mouse model of contact hypersensitivity also [21]. This effect has also been observed in pre-clinical models of cancer, where decreasing IFN- $\gamma$ expression contributes to tumor progression in mouse models of lymphoma, colorectal cancer and melanoma [22-24]. In these studies, the daily protocol for stress induction ranged from 2 to $4 \mathrm{~h}$ when restraint or immobilization was used, some other authors used psychosocial paradigms for up to $24 \mathrm{~h}$, whereas in the present study, $30 \mathrm{~min}$ of daily stress were sufficient to find a significant effect after 28 days. The relevancy of IFN- $\gamma$ suppression by stress during cancer progression should not be underestimated; however, recent findings shed light on a potential additional target of this stress-induced immune dysfunction, since it has been shown that IFN- $\gamma$ supports social behavior and neuronal connectivity [25]. Moreover, in mice knockout for IFN- $\gamma$, attenuation of the neuroendocrine and immune responses to chronic stress and disturbed spatial recognition memory in the basal state have been observed [26, 27]. These studies suggest a role for IFN-Y in mediating stress responses.

In conclusion, our results show that the daily induction in non-tumor and lymphoma bearing BALB/c mice of $30 \mathrm{~min}$ of restraint stress, with intercalated days of forced swim and days to rest, disrupts metabolism as shown by decreased weight, leptin, and insulin, this despite an absence of HPA axis activation or increasing IL-6 levels in plasma, with the potential involvement of adrenergic activation according to norepinephrine plasma levels. The protocol was also able to suppress the IFN- $\gamma$ response of lymphoma-bearing animals, a dysfunction that many studies show to be relevant to understand the relationships between neuroendocrine and immune parameters during health and disease.

\section{ACKNOWLEDGMENTS}

We are grateful to the Laboratory of Immunology and Virology, FCB, UANL for their support for this study. This work was also supported by grant CN3621504 from Programa de Investigación Científica y Tecnológica (PAICYT), Universidad Autónoma de Nuevo León (UANL), México to $\mathrm{DCH}$.

\section{DISCLOSURE}

The authors have no conflicts of interest to disclose. 


\section{REFERENCES}

1. Cao L, During MJ. What is the brain-cancer connection? Annu Rev Neurosci 2012; 35: 331-45.

2. Shin JK, Lee JY, Yang RY, et al. Molecular mechanisms underlying psychological stress and cancer. Curr Pharm Des 2016; 22: 2389-402.

3. Xie H, Li C, He Y, et al. Chronic stress promotes oral cancer growth and angiogenesis with increased circulating catecholamine and glucocorticoid levels in a mouse model. Oral Oncol 2015; 51: 991-7.

4. Buynitsky T, Mostofsky DI. Restraint stress in biobehavioral research: recent developments. Neurosci Biobehav Rev 2009; 33: 1089-98.

5. Bali A, Jaggi AS. Preclinical experimental stress studies: protocols, assessment and comparison. Eur J Pharmacol 2015; 746: 282-92.

6. Jaggi AS, Bhatia N, Kumar N, et al. A review on animal models for screening potential anti-stress agents. Neurol Sci 2011; 32: 993-1005.

7. Petit-Demouliere B, Chenu F, Bourin M. Forced swimming test in mice: a review of antidepressant activity. Psychopharmacology 2005; 177: 245-55.

8. Rohleder N, Aringer M, Boentert M. Role of interleukin-6 in stress, sleep, and fatigue. Ann N Y Acad Sci 2012; 1261: 88-96.

9. Tanaka T, Kishimoto T. Targeting interleukin-6: all the way to treat autoimmune and inflammatory diseases. Int J Biol Sci 2012; 8:1227.

10. Allen AP, Kennedy PJ, Cryan JF, et al. Biological and psychological markers of stress in humans: focus on the Trier Social Stress Test. Neurosci Biobehav Rev 2014; 38: 94-124.

11. Lee JH, Yoo SB, Kim NY, et al. Interleukin-6 and the hypothalamic-pituitary-adrenal activation in a tumor bearing mouse. Int J Neurosci 2008; 118: 355-64.

12. Kim KS, Han PL. Optimization of chronic stress paradigms using anxiety- and depression-like behavioral parameters. J Neurosci Res 2006; 83: 497-507.

13. Zamora-González EO, Santerre A, PalomeraAvalos $\mathrm{V}$, et al. A chronic combinatory stress model that activates the HPA axis and avoids habituation in BALB/C mice. J Neurosci Methods 2013; 213: 70.

14. Gomez-Flores R, Caballero-Hernandez D, TamezGuerra $\mathrm{R}$, et al. Increased survival of tumor-bearing mice by the delta opioid SNC 80. Anticancer Res 2005; 25: 4563-7.

15. Chiba $S$, Numakawa $T$, Ninomiya $M$, et al. Chronic restraint stress causes anxiety- and depression-like behaviors, downregulates glucocorticoid receptor expression, and attenuates glutamate release induced by brain-derived neurotrophic factor in the prefrontal cortex. Prog Neuropsychopharmacol Biol Psychiatry 2012; 39: 112-9.

16. Strekalova T, Spanagel R, Bartsch D, et al. Stressinduced anhedonia in mice is associated with deficits in forced swimming and exploration. Neuropsychopharmacology 2004; 29: 2007-17.

17. Otovic $P$, Hutchinson E. Limits to using HPA axis activity as an indication of animal welfare. Altex 2015; 32: 41-50.

18. Li G, Klein RL, Matheny M, et al. Induction of uncoupling protein 1 by central interleukin- 6 gene delivery is dependent on sympathetic innervation of brown adipose tissue and underlies one mechanism of body weight reduction in rats. Neuroscience 2002; 115: 879-89.

19. Himmerich H, Fischer J, Bauer K, et al. Stress-induced cytokine changes in rats. Eur Cytokine Netw 2013; 24: 97-103.

20. Kalinichenko LS, Koplik EV, Pertsov SS. Cytokine profile of peripheral blood in rats with various behavioral characteristics during acute emotional stress. Bull Exp Biol Med 2014; 156: 441.

21. Hall JM, Witter AR, Racine RR, et al. Chronic psychological stress suppresses contact hypersensitivity: Potential roles of dysregulated cell trafficking and decreased IFN- $\gamma$ production. Brain Behav Immun 2014; 36: 156-64.

22. Frick LR, Barreiro Arcos ML, Rapanelli M, et al. Chronic restraint stress impairs T-cell immunity and promotes tumor progression in mice. Stress 2009; 12: 134-43.

23. Peters $S$, Grunwald $N$, Rümmele $P$, et al. Chronic psychosocial stress increases the risk for inflammation-related colon carcinogenesis in male mice. Stress 2012; 15: 403-15.

24. Sommershof A, Scheuermann L, Koerner J, et al. Chronic stress suppresses anti-tumor T CD8+ responses and tumor regression following cancer immunotherapy in a mouse model of melanoma. Brain Behav Immun 2017; 65: $140-9$.

25. Filiano AJ, Xu Y, Tustison NJ, et al. Unexpected role of interferon- $\gamma$ in regulating neuronal connectivity and social behaviour. Nature 2016; 535: 425-9.

26. Litteljohn D, Cummings A, Brennan A, et al. Interferon-gamma deficiency modifies the effects of a chronic stressor in mice: implications for psychological pathology. Brain Behav Immun 2010; 24: 462-73.

27. Litteljohn D, Nelson E, Hayley S. IFN- $\gamma$ differentially modulates memory-related processes under basal and chronic stressor conditions. Front Cell Neurosci 2014; 8: 391. 